\title{
Comparative Evaluation of Hyoid Bone Position and Tongue Area with Twin Block and forsus: A Randomized Clinical Trial
}

\author{
Dr Raksha Rajput,' Dr Suchita Daokar² \\ 'Senior lecturer, Dept of Orthodontics, YCDC Dental College, Maharashtra, India; \\ 2Professor, PG \& PhD guide, Dept of Orthodontics, CSMSS Dental College, Maharashtra, India \\ Correspondence: Dr Raksha Rajput; Email: rajputraksha6@gmail.com
}

\section{ABSTRACT}

Introduction: Class II div 1 malocclusion is most commonly presented with retruded mandible. This backward placement of mandible pushes the tongue posteriorly and inturn impairs the position of hyoid bone and total tongue area. Correction of retruded mandible with functional appliances is also considered to have positive effect on hyoid bone position and tongue area. Aim and Objectives: The objective of this study was to evaluate and compare the effects of Twin Block and Forsus on the hyoid bone position and tongue area in the treatment of Class II division 1 malocclusion.

Materials \& Method: A 2-arm parallel, randomized controlled trial was designed consisting of 24 Class II division 1 malocclusion patients indicated for treatment with functional appliances. 24 patients were randomized and equally divided among Twin Block (Group A) and Forsus (Group B) group. Pre- and post functional lateral cephalograms of both groups were traced and statistically analysed using paired t-test and T test of Equality of Means. ' $p$ ' value of less than 0.05 was considered as statistically significant.

Result: A significantly upward and forward movement of hyoid bone was found with both the appliances. Tongue area also improved with Twin Block and Forsus group. On intergroup comparison insignificant results were observed between both the appliances indicating similar effect on tongue area and hyoid bone position.

Conclusion: Twin Block has more skeletal effect than Forsus, still both Twin Block and Forsus are effective in improving tongue area and forward and upward displacement of hyoid bone position while correcting Class II malocclusion.

Keywords: Class II division 1 malocclusion, Forsus, Hyoid bone, Tongue area, Twin Block.

\section{INTRODUCTION}

Class II malocclusion is the most commonly encountered problem in orthodontic practice.' Mandibular retrusion is the main etiological factor in class II subjects.

Najda ${ }^{2}$ showed that individuals with mandibular Class II malocclusion have upper airways measurements diminishedindicating a correlationbetweenmandibular length and position and the size of oropharynx and nasopharynx. Backward placement of mandible pushes the tongue posteriorly, decreasing the total available space for tongue and pharyngeal airway space which may in turn impair respiratory functions during day and also cause nocturnal problems such as snoring, Upper airway resistance syndrome (UARS) and obstructive sleep apnea syndrome (OSAS). ${ }^{3}$ Enache $^{4}$ found the significant positive correlation between $\mathrm{AHI}$ and ANB suggesting that the patients with OSA had a retrognathic sagittal pattern with a class II anteroposterior relationship.

Khannna $^{5}$ study on Angle's Class \|l division I malocclusion with retrognathic mandible showed an inferoposterior displacement of hyoid bone and the position alteration was prevalent in skeletal malrelationship rather than dento-alveolar malocclusion. Johal investigated that the hyoid bone is more inferiorly positioned in OSA patient. The hyoid would tend to pull the tongue backwards, further narrowing the pharyngeal airway and thus could form a prognostic indicator for OSA severity.

Hence, this study was designed to compare the changes in skeletal, hyoid bone position and tongue area with Twin Block and Forsus. 


\section{MATERIALS AND METHOD}

The present study was a prospective, double-blind, randomized clinicalstudy conducted in the Department of Orthodontics and Dentofacial Orthopedics, CSMSS Dental college and hospital, Aurangabad.

Sample size was calculated with a type 1 error frequency of $5 \%$ and power of the statistical test set at $80 \%$. 12 patients were enrolled in each group.

Out of 94 patients screened from the OPD, 45 patients were selected based on clinical examinations, which were further sent for radiographic investigations. The inclusion criteria were growing (CVMI stages till 5) subjects with skeletal Class II division 1 malocclusion exhibiting overjet of 4-10 mm, retrognathic mandible, horizontal growth pattern, with complete set of permanent dentition excluding third molars displaying positive VTO (Subjects not meeting the inclusive criteria were excluded). On assessing lateral cephalograms of the 45 subjects, 21 patients not meeting the inclusion criteria were further excluded. The study sample consisted of 24 patients.

\section{In Vivo Study}

The lateral cephalograms of the subjects were categorized into the following groups (gender and age equity)-

Group A (Twin block group) \& Group B (Forsus group). Each group consisted of 12 subjects.

\section{Randomization and Allocation Concealment}

Once informed consent had been obtained, subjects were allocated to Group A or Group B using a block randomization determined by a computer-generated random number table. Block sizes of 2, 4, 6 were used within each group. The sequence of the block sizes generated by the computer was 4, 6 then 2 . In the first block, they were numbered from 1 to 4 , in the second block they were numbered 1 to 6 and in the third block from 1 to 2. Based on the randomisation list generated they were allocated to either group A or group B. Same list were used to allocate the female participants to the group.

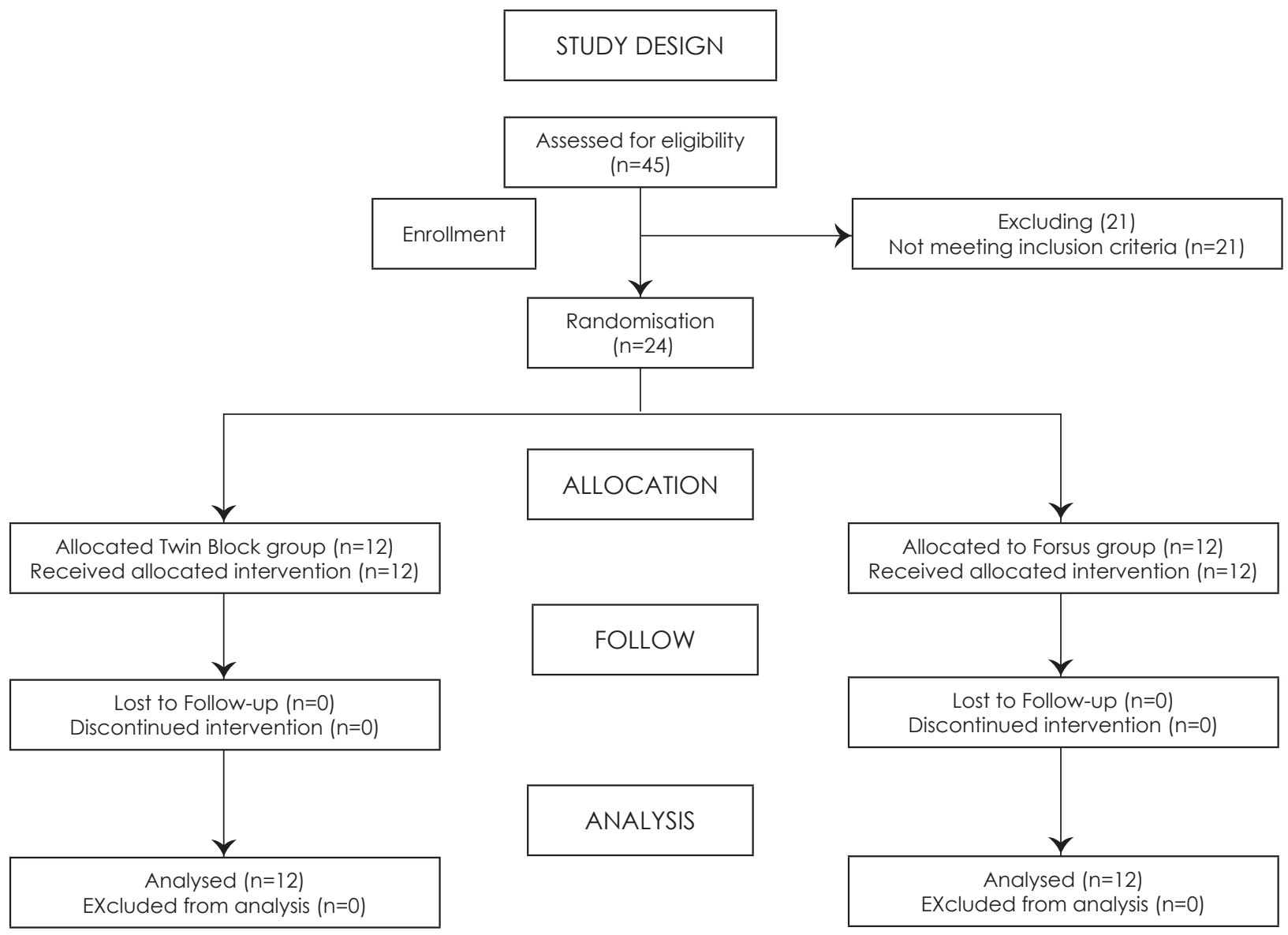

Figure 1: Consort Form 


\section{Interventions}

All of the participants in both treatment groups were treated by the principal investigator with Twin Block and Forsus appliance.

A. Bite registration for construction of Twin block appliance (Group A patients).

The conventional twin block appliance with a construction bite of edge-to-edge incisal relationship was delivered with all the instructions to the patients.

B. Fixed orthodontic treatment for installation of Forsus appliance in Group B patients.

Group B subjects underwent a specific treatment protocol with MBT prescription with an 0.022 slot preadjusted fixed appliances in combination with the FRD. After aligning and leveling phase, a $0.019 \times 0.025$-inch SS arch wire was inserted in both arches. A transpalatal arch in the upper arch to control the transverse expansion of maxillary first molars and lingual crown torque of $10^{\circ}$ in the lower anterior segment was placed to minimize flaring caused by the fixed functional appliances. Also the mandibular archwire was cinched distal to molars. The FRD was engaged and continued until the Class II were corrected to edge to edge incisor relationship.

The patients were observed at 4-week intervals for a period of 6 months and appliances were activated as needed.

\section{Cephalometric Analysis}

Lateral cephalometric radiographs were taken before starting (T1) and after removal (T2) of the functional appliance therapy on the same machine with standardized head position and were traced and analyzed manually by the same operator. The readings were taken thrice and mean of which are taken so as to overcome any tracing errors.

\section{Blinding}

Blinding of participants in each group was done. As the primary investigator who performed the procedure could not be blinded, so both the co investigator who analyzed pre- and post functional lateral cephalograms of both groups and the statistician were blinded with regard to the group to which each lateral cephalogram belonged.

The cephalometric landmarks and lines used to assess the changes in hyoid position, and tongue area are shown in Table \& Figure no 2.

I. Skeletal tissue analysis-
1. SNA angle

2. SNB angle

3. Effective maxillary length

4. Effective mandibular length

5. W angle

6. FMPA (Frankforts Mandibular Plane angle)

II. Hyoid bone position.

Horizontal changes in the hyoid bone position were determined by

1. Hy-aC2, the linear distance between Hy and $\mathrm{aC2}$.

2. Hy-aC3, the linear distance between Hy and aC3.

Vertical changes in the hyoid bone position were determined by

3. Hy-NL, the perpendicular distance from NSL to hyoid.

4. Hy-MP, the perpendicular distance from MP to hyoid.

III. Tongue area.

Area enclosed posteriorly by the oropharynx and uvula, superiorly by the hard palate, and anteriorly by the lingual aspects of the anterior teeth and lingual mandibular symphyseal contour. The inferior border is the line extending from the vallecula to the most anterior point on the hyoid body and the line from the most anterior point on the hyoid bone to the menton (Figure 2). The tongue area has no definite shape and being an irregular shape, is measured using 'offset method'.

The method is as follows: Length of the longest axis of the area is measured (I). Next, divide the length line into equal sections. At each of these point, measure the distance across the area in a line perpendicular to the length line at each point (through a to e). These lines are offset line. Finally, add the length of all offset lines and multiply the result times the distance that separate these lines.

\section{Statistical Analysis}

Statistical analyses were performed with software package SPSS (for Windows 7, version 16.0, SPSS). Pre versus post treatment values were analyzed and mean, standard deviation and paired t-test was carried. Intergroup comparison of various parameters was performed using T test of Equality of Means.

A ' $p$ ' value of less than 0.05 was considered as statistically significant. 

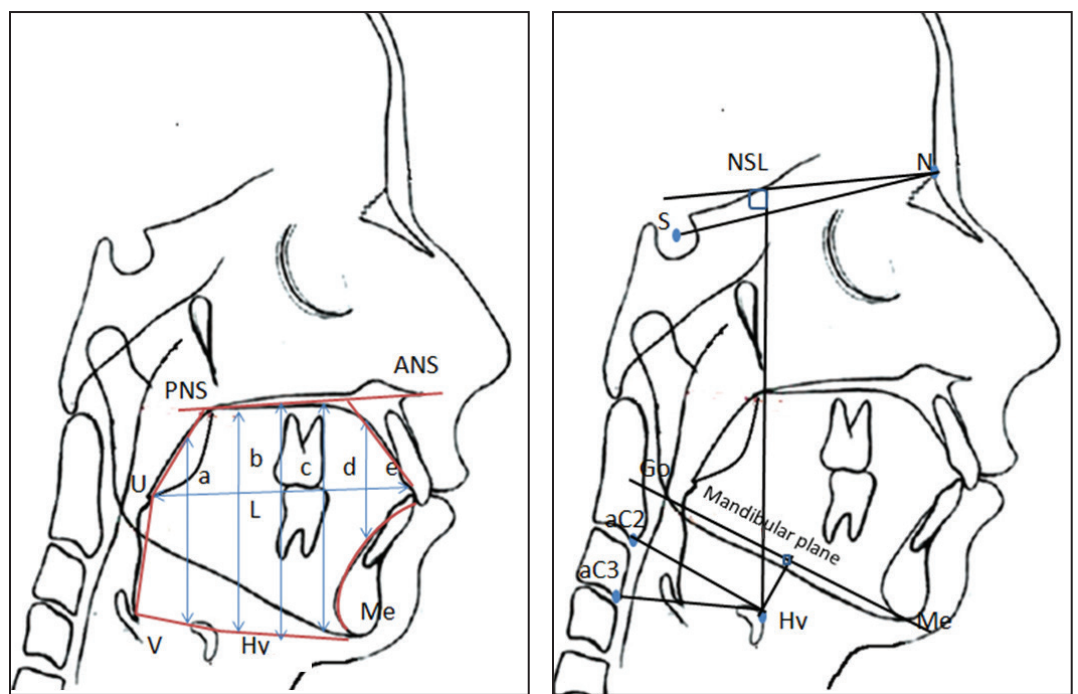

Figure 2: Cephalometric Landmarks used for measuring Hyoid bone position and Tongue area.
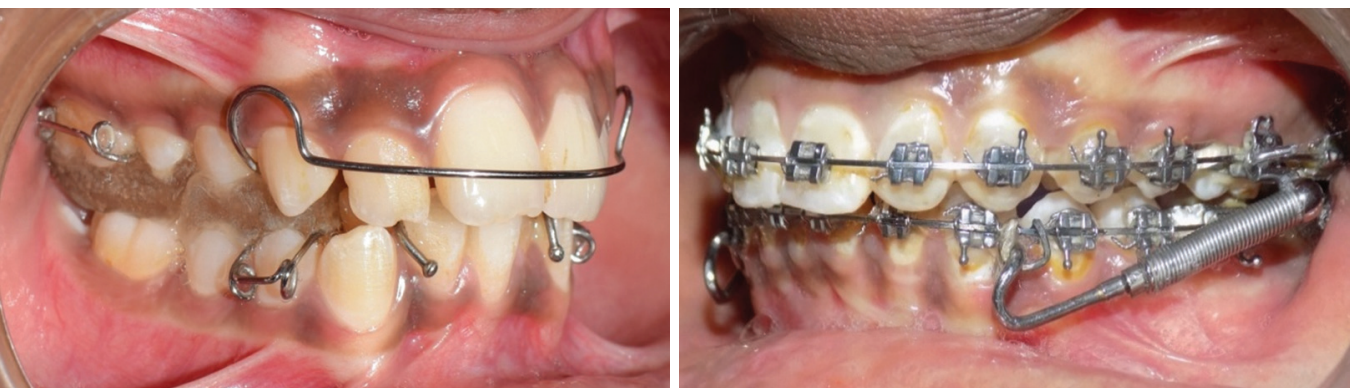

Figure 3: Twin Block and Forsus appliance installation

\section{RESULT}

In Skeletal parameters, statistically significant increase was seen with SNB angle from $74.08^{\circ}$ to $76.92^{\circ}(p=0.000)$, effective mandibular length from $93.25 \mathrm{~mm}$ to $97.42 \mathrm{~mm}$ $(p=0.000), W$ angle from 48.50 to $51.75(p=0.000)$ FMP angle from $20.00^{\circ}$ to $22.67^{\circ}(p=0.000)$ in group $A$. However, SNA angle showed statistically significant decrease from $80.58^{\circ}$ to $79.17^{\circ}(p=0.000)$ and effective maxillary length ( $p=0.012$ ) (Table no. 1\&2).

Similarly in group B significant increase in SNB angle from $73.67^{\circ}$ to $74.58^{\circ}(p=0.001)$, effective mandibular length from $102.58 \mathrm{~mm}$ to $104.58 \mathrm{~mm}(p=0.001), W$ angle from $50.00^{\circ}$ to $51.67^{\circ}$ ( $\left.p=0.000\right)$, FMP angle from $20.75^{\circ}$ to $22.75^{\circ}$ ( $p=0.000$ ) was observed. No significant difference was seen in SNA angle $(p=0.586)$, effective maxillary length $(p=0.674)$ after the treatment.

Inter group comparison between Twin Block and Forsus showed significant difference with SNA $(p=0.001)$, SNB angle $(p=0.000)$, effective mandibular length $(p=0.004)$ and YEN angle $(p=0.000)$ indicating Twin Block having greater skeletal changes than Forsus whereas no significant results were found with effective maxillary length $(p=0.501)$, FMP angle $(p=0.152)$.
Significant differences were found in the position of hyoid bone and tongue area from pre to post treatment [Table no.II-1 (B) \& III-1 (B)]. When hyoid bone position was measured with vertical linear measurements, a statistically significant upward movement of hyoid bone in both Group A \& B determined by change in values in Hy-NL $(p=0.003), \quad(p=0.043)$ from $92.33 \mathrm{~mm}$ to $89.33 \mathrm{~mm} ; 106.83 \mathrm{~mm}$ to $105.42 \mathrm{~mm}$ and $\operatorname{Hy}-\mathrm{MP}(\mathrm{p}=$ $0.013) ;(p=0.001)$ from $9.92 \mathrm{~mm}$ to $7.50 \mathrm{~mm} ; 10.83 \mathrm{~mm}$ to $9.58 \mathrm{~mm}$ respectively. In horizontal dimensions the forward movement of hyoid bone determined by $\mathrm{Hy}-\mathrm{aC} 2$ and $\mathrm{Hy}-\mathrm{aC} 3$ was also found significant $(p=0.050) ;(p=0.000) \quad \&(p=0.003) ;(p=0.034)$ in both groups.

The inter-group comparison between the effect of two appliance, revealed insignificant $(p=0.087),(p=0.119)$, $(p=0.164),(p=0.101)$ results, showing similar movement of hyoid bone with both appliances.

The tongue area increased significantly with the Twin Block and Forsus ( $p=0.003),(p=0.014)$ from $14435.33 \mathrm{~mm}^{2}$ to $15562.25 \mathrm{~mm}^{2}$ and $19423.58 \mathrm{~mm}^{2}$ to $20162.75 \mathrm{~mm}^{2}$ respectively, the intergroup showed insignificant $(p=0.330)$ difference between both the appliances, indicating similar effect on tongue area. 
Table I-1(A): Pre \& post treatment values of Skeletal Parameters after using Twin Block (Group A) and Forsus (Group B)

\begin{tabular}{|c|c|c|c|c|c|c|}
\hline Parameter & Group & Value & Mean & $\mathbf{N}$ & Std. Deviation & Std. Error Mean \\
\hline \multirow{4}{*}{ SNA Angle } & \multirow{2}{*}{ Twin Block } & Pre & 80.58 & 12 & 2.065 & 0.596 \\
\hline & & Post & 79.17 & 12 & 1.946 & 0.562 \\
\hline & \multirow{2}{*}{ Forsus } & Pre & 79.08 & 12 & 1.676 & 0.484 \\
\hline & & Post & 79.17 & 12 & 1.697 & 0.490 \\
\hline \multirow{4}{*}{ SNB Angle } & \multirow{2}{*}{ Twin Block } & Pre & 74.08 & 12 & 2.539 & 0.733 \\
\hline & & Post & 76.92 & 12 & 2.539 & 0.733 \\
\hline & \multirow{2}{*}{ Forsus } & Pre & 73.67 & 12 & 3.367 & 0.972 \\
\hline & & Post & 74.58 & 12 & 3.288 & 0.949 \\
\hline \multirow{4}{*}{$\begin{array}{l}\text { Effective Maxillary } \\
\text { Length }\end{array}$} & \multirow{2}{*}{ Twin Block } & Pre & 79.67 & 12 & 6.933 & 2.001 \\
\hline & & Post & 79.08 & 12 & 7.038 & 2.032 \\
\hline & \multirow{2}{*}{ Forsus } & Pre & 85.08 & 12 & 7.141 & 2.061 \\
\hline & & Post & 85.17 & 12 & 7.120 & 2.055 \\
\hline \multirow{4}{*}{$\begin{array}{l}\text { Effective Mandibular } \\
\text { Length }\end{array}$} & \multirow{2}{*}{ Twin Block } & Pre & 93.25 & 12 & 8.433 & 2.434 \\
\hline & & Post & 97.42 & 12 & 8.393 & 2.423 \\
\hline & \multirow{2}{*}{ Forsus } & Pre & 102.58 & 12 & 5.616 & 1.621 \\
\hline & & Post & 104.58 & 12 & 5.616 & 1.621 \\
\hline \multirow{4}{*}{ W Angle } & \multirow{2}{*}{ Twin Block } & Pre & 48.50 & 12 & 2.067 & 0.597 \\
\hline & & Post & 51.75 & 12 & 1.765 & 0.509 \\
\hline & \multirow{2}{*}{ Forsus } & Pre & 50.00 & 12 & 2.558 & 0.739 \\
\hline & & Post & 51.67 & 12 & 2.229 & 0.644 \\
\hline \multirow{4}{*}{ FMPA Angle } & \multirow{2}{*}{ Twin Block } & Pre & 20.00 & 12 & 4.221 & 1.219 \\
\hline & & Post & 22.67 & 12 & 3.725 & 1.075 \\
\hline & \multirow{2}{*}{ Forsus } & Pre & 20.75 & 12 & 2.633 & 0.760 \\
\hline & & Post & 22.75 & 12 & 2.667 & 0.770 \\
\hline
\end{tabular}

Table I-1(B): Twin Block (Group A) and Forsus (Group B) Pre vs. Post treatment comparison (paired t-test)

\begin{tabular}{|c|c|c|c|c|c|c|c|c|c|c|}
\hline \multirow{3}{*}{\multicolumn{2}{|c|}{ Parameter }} & \multicolumn{5}{|c|}{ Paired Differences } & \multirow{3}{*}{-} & \multirow{3}{*}{ ד } & \multirow{3}{*}{$\begin{array}{l}\text { ò } \\
\frac{0}{\overline{0}} \\
\frac{1}{\dot{1}} \\
\frac{0}{n}\end{array}$} & \multirow{3}{*}{ 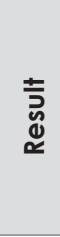 } \\
\hline & & \multirow{2}{*}{ c } & \multirow{2}{*}{ 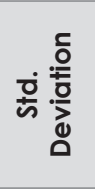 } & \multirow{2}{*}{ 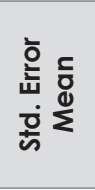 } & \multicolumn{2}{|c|}{$\begin{array}{l}95 \% \text { Confidence Interval } \\
\text { of the Difference }\end{array}$} & & & & \\
\hline & & & & & 㐫 & $\begin{array}{l}\text { ळ } \\
\text { 음 }\end{array}$ & & & & \\
\hline \multirow{2}{*}{ SNA Angle } & Twin Block & 1.417 & 0.996 & 0.288 & 0.784 & 2.050 & 4.926 & 11 & 0.000 & S \\
\hline & Forsus & -0.083 & 0.515 & 0.149 & -0.411 & 0.244 & -0.561 & 11 & 0.586 & NS \\
\hline \multirow{2}{*}{ SNB Angle } & Twin Block & -2.833 & 1.030 & 0.297 & -3.488 & -2.179 & -9.530 & 11 & 0.000 & $S$ \\
\hline & Forsus & -0.917 & 0.669 & 0.193 & -1.341 & -0.492 & -4.750 & 11 & 0.001 & S \\
\hline \multirow{2}{*}{$\begin{array}{l}\text { Effective } \\
\text { Maxillary Length }\end{array}$} & Twin Block & 0.583 & 0.669 & 0.193 & 0.159 & 1.008 & 3.023 & 11 & 0.012 & $S$ \\
\hline & Forsus & -0.083 & 0.669 & 0.193 & -0.508 & 0.341 & -0.432 & 11 & 0.674 & NS \\
\hline \multirow{2}{*}{$\begin{array}{l}\text { Effective } \\
\text { Mandibular } \\
\text { Length }\end{array}$} & Twin Block & -4.167 & 1.697 & 0.490 & -5.245 & -3.089 & -8.507 & 11 & 0.000 & S \\
\hline & Forsus & -2.000 & 1.651 & 0.477 & -3.049 & -0.951 & -4.195 & 11 & 0.001 & $S$ \\
\hline \multirow{2}{*}{ W Angle } & Twin Block & -3.250 & 1.055 & 0.305 & -3.920 & -2.580 & -10.668 & 11 & 0.000 & $S$ \\
\hline & Forsus & -1.667 & 0.985 & 0.284 & -2.292 & -1.041 & -5.863 & 11 & 0.000 & $S$ \\
\hline \multirow{2}{*}{ FMPA Angle } & Twin Block & -2.667 & 0.985 & 0.284 & -3.292 & -2.041 & -9.381 & 11 & 0.000 & S \\
\hline & Forsus & -2.000 & 1.206 & 0.348 & -2.766 & -1.234 & -5.745 & 11 & 0.000 & $S$ \\
\hline
\end{tabular}


Table II-1(A): Pre \& post treatment values of Hyoid Bone position after using Twin Block (Group A) and Forsus (Group B)

\begin{tabular}{|c|c|c|c|c|c|c|}
\hline Parameter & Group & Values & Mean & $\mathbf{N}$ & Std. Deviation & Std. Error Mean \\
\hline \multirow{4}{*}{$\mathrm{Hy}-\mathrm{NL}$} & \multirow{2}{*}{ Twin Block } & Pre & 92.33 & 12 & 9.875 & 2.851 \\
\hline & & Post & 89.33 & 12 & 9.773 & 2.821 \\
\hline & \multirow{2}{*}{ Forsus } & Pre & 106.83 & 12 & 8.167 & 2.358 \\
\hline & & Post & 105.42 & 12 & 7.879 & 2.275 \\
\hline \multirow{4}{*}{ Ну-МР } & \multirow{2}{*}{ Twin Block } & Pre & 9.92 & 12 & 4.795 & 1.384 \\
\hline & & Post & 7.50 & 12 & 4.543 & 1.311 \\
\hline & \multirow{2}{*}{ Forsus } & Pre & 10.83 & 12 & 4.988 & 1.440 \\
\hline & & Post & 9.58 & 12 & 4.870 & 1.406 \\
\hline \multirow{4}{*}{ Hy-aC2 } & \multirow{2}{*}{ Twin Block } & Pre & 34.42 & 12 & 3.728 & 1.076 \\
\hline & & Post & 34.42 & 12 & 4.100 & 1.184 \\
\hline & \multirow{2}{*}{ Forsus } & Pre & 32.42 & 12 & 3.728 & 1.076 \\
\hline & & Post & 34.42 & 12 & 4.100 & 1.184 \\
\hline \multirow{4}{*}{ Hy-aC3 } & \multirow{2}{*}{ Twin Block } & Pre & 28.42 & 12 & 3.397 & 0.981 \\
\hline & & Post & 30.17 & 12 & 3.433 & 0.991 \\
\hline & \multirow{2}{*}{ Forsus } & Pre & 29.67 & 12 & 3.798 & 1.096 \\
\hline & & Post & 30.58 & 12 & 4.209 & 1.215 \\
\hline
\end{tabular}

Table II-1(B): Twin Block (Group A) and Forsus (Group B) Pre vs. Post treatment comparison (paired t-test)

\begin{tabular}{|c|c|c|c|c|c|c|c|c|c|c|}
\hline \multirow{3}{*}{$\begin{array}{l}\frac{\bar{d}}{0} \\
\frac{\varepsilon}{0} \\
\overline{0}\end{array}$} & \multirow{3}{*}{$\frac{0}{\frac{0}{2}}$} & \multicolumn{5}{|c|}{ Paired Differences } & \multirow{3}{*}{-} & \multirow{3}{*}{ ॅั } & \multirow{3}{*}{ 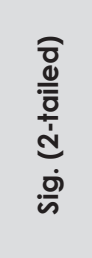 } & \multirow{3}{*}{$\begin{array}{l}\bar{\nu} \\
\stackrel{\bar{g}}{\alpha}\end{array}$} \\
\hline & & \multirow{2}{*}{ 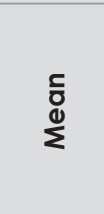 } & \multirow{2}{*}{ 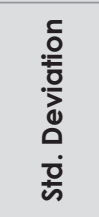 } & \multirow{2}{*}{ 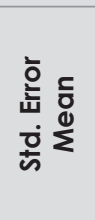 } & \multicolumn{2}{|c|}{$\begin{array}{l}95 \% \text { Confidence } \\
\text { Interval of the } \\
\text { Difference }\end{array}$} & & & & \\
\hline & & & & & 㐫 & $\begin{array}{l}\bar{\varpi} \\
\frac{0}{0} \\
\frac{2}{2}\end{array}$ & & & & \\
\hline \multirow{2}{*}{$\mathrm{Hy}-\mathrm{NL}$} & Twin Block & 3.000 & 2.697 & 0.778 & 1.287 & 4.713 & 3.854 & 11 & 0.003 & $S$ \\
\hline & Forsus & 1.417 & 2.151 & 0.621 & 0.050 & 2.784 & 2.281 & 11 & 0.043 & $S$ \\
\hline \multirow{2}{*}{ Hy-MP } & Twin Block & 2.417 & 2.843 & 0.821 & 0.610 & 4.223 & 2.945 & 11 & 0.013 & $S$ \\
\hline & Forsus & 1.250 & 0.965 & 0.279 & 0.637 & 1.863 & 4.486 & 11 & 0.001 & $S$ \\
\hline \multirow{2}{*}{ Hy-aC2 } & Twin Block & -1.583 & 2.539 & 0.733 & -3.197 & 0.030 & -2.160 & 11 & 0.050 & $S$ \\
\hline & Forsus & -2.000 & 1.348 & 0.389 & -2.857 & -1.143 & -5.138 & 11 & 0.000 & $S$ \\
\hline \multirow{2}{*}{$\mathrm{Hy}-\mathrm{aC} 3$} & Twin Block & -1.750 & 1.603 & 0.463 & -2.768 & -0.732 & -3.783 & 11 & 0.003 & $S$ \\
\hline & Forsus & -0.917 & 1.311 & 0.379 & -1.750 & -0.083 & -2.421 & 11 & 0.034 & $S$ \\
\hline
\end{tabular}

Table III-1(A): Pre \& post treatment values of Tongue area after using Twin Block (Group A) and Forsus (Group B)

\begin{tabular}{|c|c|c|c|c|c|c|}
\hline \multirow{1}{*}{ Parameter } & Group & Values & Mean & N & Std. Deviation & Std. Error Mean \\
\hline \multirow{3}{*}{ Tongue Area } & \multirow{2}{*}{ Twin Block } & Pre & 14435.33 & 12 & 2970.589 & 857.535 \\
\cline { 2 - 8 } & & Post & 15562.25 & 12 & 3136.490 & 905.427 \\
\cline { 2 - 8 } & \multirow{2}{*}{ Forsus } & Pre & 19423.58 & 12 & 2391.652 & 690.411 \\
\cline { 2 - 8 } & \multirow{2}{*}{$*$} & Post & 20162.75 & 12 & 2541.128 & 733.560 \\
\hline
\end{tabular}

Table III-1(B): Twin Block (Group A) and Forsus (Group B) Pre vs. Post treatment comparison (paired t-test)

\begin{tabular}{|c|c|c|c|c|c|c|c|c|c|c|}
\hline \multirow{3}{*}{ 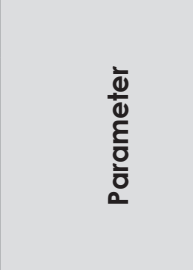 } & \multirow{3}{*}{$\begin{array}{l}\frac{0}{2} \\
\frac{0}{0}\end{array}$} & \multicolumn{5}{|c|}{ Paired Differences } & \multirow{3}{*}{-} & \multirow{3}{*}{$\overleftarrow{7}$} & \multirow{3}{*}{ 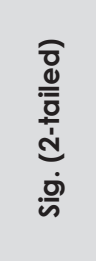 } & \multirow{3}{*}{ 声 } \\
\hline & & \multirow{2}{*}{ ర్ } & \multirow{2}{*}{ 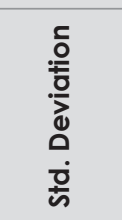 } & \multirow{2}{*}{ 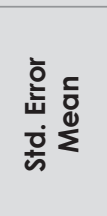 } & \multicolumn{2}{|c|}{$\begin{array}{l}95 \% \text { Confidence } \\
\text { Interval of the } \\
\text { Difference }\end{array}$} & & & & \\
\hline & & & & & 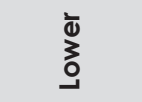 & $\begin{array}{l}\bar{\Phi} \\
\frac{0}{2}\end{array}$ & & & & \\
\hline \multirow{2}{*}{ Tongue Area } & Twin Block & -1126.91 & 1034.25 & 298.56 & -1784.04 & -469.78 & -3.77 & 11 & 0.003 & S \\
\hline & Forsus & -739.16 & 881.71 & 254.52 & -1299.38 & -178.95 & -2.90 & 11 & 0.014 & S \\
\hline
\end{tabular}


Table I-2(A): Twin Block vs. Forsus (Group A vs. Group B) comparison of mean difference of Skeletal parameter (pre and post treatment mean) values.

\begin{tabular}{|l|c|c|c|c|c|}
\hline \multicolumn{1}{|c|}{ Parameter } & Group & N & Mean & Std. Deviation & Std. Error Mean \\
\hline \multirow{3}{*}{ SNA Angle } & Twin Block & 12 & 1.42 & 0.996 & 0.288 \\
\cline { 2 - 6 } & Forsus & 12 & 0.25 & 0.452 & 0.131 \\
\hline \multirow{2}{*}{ SNB Angle } & Twin Block & 12 & 2.83 & 1.030 & 0.297 \\
\cline { 2 - 6 } & Forsus & 12 & 0.92 & 0.669 & 0.193 \\
\hline \multirow{2}{*}{$\begin{array}{l}\text { Effective Maxillary } \\
\text { Length }\end{array}$} & Twin Block & 12 & 0.58 & 0.669 & 0.193 \\
\hline \multirow{2}{*}{$\begin{array}{l}\text { Effective Mandibular } \\
\text { Length }\end{array}$} & Forsus & 12 & 0.42 & 0.515 & 0.149 \\
\hline \multirow{2}{*}{ W Angle } & Twin Block & 12 & 4.17 & 1.697 & 0.490 \\
\cline { 2 - 6 } & Forsus & 12 & 2.00 & 1.651 & 0.477 \\
\hline \multirow{2}{*}{ FMPA Angle } & Twin Block & 12 & 3.25 & 1.055 & 0.305 \\
\cline { 2 - 6 } & Forsus & 12 & 2.67 & 0.985 & 0.284 \\
\hline
\end{tabular}

Table I-2(B): Twin Block vs. Forsus (Group A vs. Group B) comparison using t-test of Equality of Means

\begin{tabular}{|l|c|c|c|c|c|c|}
\hline \multirow{2}{*}{\multicolumn{1}{|c|}{ Parameter }} & \multicolumn{5}{c|}{ t-test for Equality of Means } & \multirow{2}{*}{ Result } \\
\cline { 2 - 7 } & T & df & Sig. (2-tailed) & Mean Difference & Std. Error Difference \\
\hline SNA Angle & 3.694 & 22 & 0.001 & 1.167 & 0.316 & S \\
\hline SNB Angle & 5.408 & 22 & 0.000 & 1.917 & 0.354 & S \\
\hline Effective Maxillary Length & .684 & 22 & 0.501 & 0.167 & 0.244 & NS \\
\hline Effective Mandibular Length & 3.170 & 22 & 0.004 & 2.167 & 0.683 & S \\
\hline W Angle & 3.800 & 22 & 0.001 & 1.583 & 0.449 & S \\
\hline FMPA Angle & 1.483 & 22 & 0.152 & 0.667 & NS \\
\hline
\end{tabular}

Table II-2(A): Twin Block vs. Forsus (Group A vs. Group B) comparison of mean difference of Hyoid Bone position (pre and post treatment mean) values.

\begin{tabular}{|c|c|c|c|c|c|}
\hline \multicolumn{2}{|c|}{ Group } & $\mathbf{N}$ & Mean & Std. Deviation & Std. Error Mean \\
\hline \multirow{2}{*}{ Hy-NL } & Twin Block & 12 & -3.00 & 2.697 & 0.778 \\
\hline & Forsus & 12 & -1.42 & 1.443 & 0.417 \\
\hline \multirow{2}{*}{ Hy-MP } & Twin Block & 12 & -2.58 & 2.678 & 0.773 \\
\hline & Forsus & 12 & -1.25 & 0.965 & 0.279 \\
\hline \multirow{2}{*}{ Hy-aC2 } & Twin Block & 12 & 1.58 & 2.539 & 0.733 \\
\hline & Forsus & 12 & 0.33 & 1.614 & 0.466 \\
\hline \multirow{2}{*}{ Hy-aC3 } & Twin Block & 12 & 1.75 & 1.603 & 0.463 \\
\hline & Forsus & 12 & 0.58 & 1.730 & 499.000 \\
\hline
\end{tabular}

Table II-2(B): Twin Block vs. Forsus (Group A vs. Group B) comparison using t-test of Equality of Means

\begin{tabular}{|c|c|c|c|c|c|c|}
\hline \multirow{2}{*}{ Parameter } & \multicolumn{5}{|c|}{ t-test for Equality of Means } & \multirow{2}{*}{ Result } \\
\hline & $T$ & df & Sig. (2-tailed) & Mean Difference & Std. Error Difference & \\
\hline Hy-NL & -1.793 & 22 & 0.087 & -1.583 & 0.883 & NS \\
\hline Hy-MP & -1.622 & 22 & 0.119 & -1.333 & 0.822 & NS \\
\hline Hy-aC2 & 1.439 & 22 & 0.164 & 1.250 & 0.869 & NS \\
\hline $\mathrm{Hy}-\mathrm{aC} 3$ & 1.714 & 22 & 0.101 & 1.167 & 0.681 & NS \\
\hline
\end{tabular}


Table III-2(A): Twin Block vs. Forsus (Group A vs. Group B) comparison of mean difference of Tongue area (pre and post treatment mean) values.

\begin{tabular}{|l|c|c|c|c|c|}
\hline \multicolumn{2}{|c|}{ Group } & N & Mean & Std. Deviation & Std. Error Mean \\
\hline \multirow{2}{*}{ Tongue Area } & Twin Block & 12 & 1129.92 & 1033.316 & 298.293 \\
\cline { 2 - 7 } & Forsus & 12 & 739.17 & 881.716 & 254.529 \\
\hline
\end{tabular}

Table III-2(B): Twin Block vs. Forsus (Group A vs. Group B) comparison using t-test of Equality of Means

\begin{tabular}{|l|c|c|c|c|c|c|}
\hline \multirow{2}{*}{\multicolumn{1}{|c|}{ Parameter }} & \multicolumn{5}{|c|}{ t-test for Equality of Means } \\
\cline { 2 - 7 } & T & df & Sig. (2-tailed) & Mean Difference & Std. Error Difference & Result \\
\hline Tongue area & .996 & 22 & 0.330 & 390.750 & 392.127 & NS \\
\hline
\end{tabular}

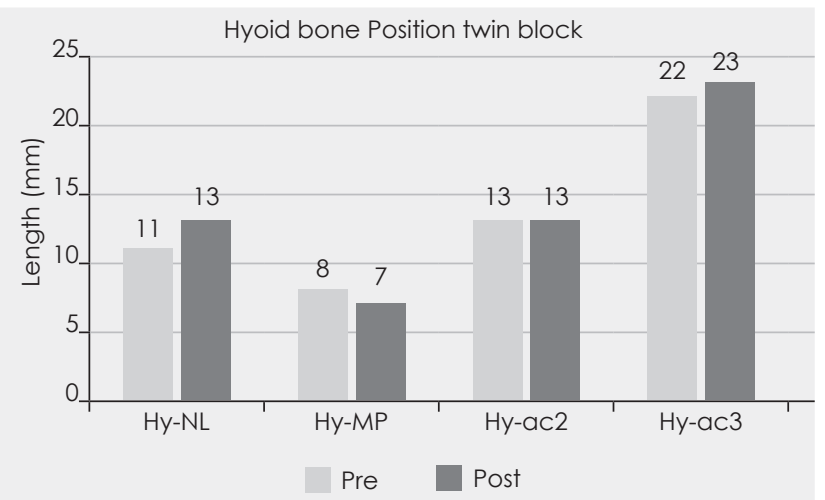

Graph 1: Comparison of mean of Hyoid Bone position in pre and post treatment of Twin Block (Group A)

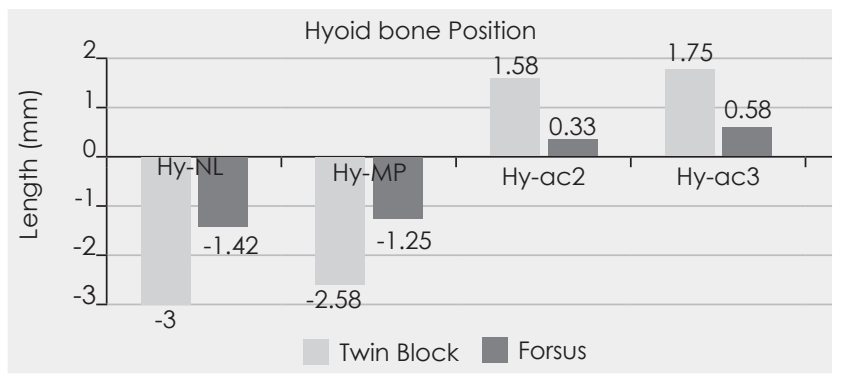

Graph 3: Twin Block (Group A) vs. Forsus (Group B) comparison of mean difference of Hyoid Bone position

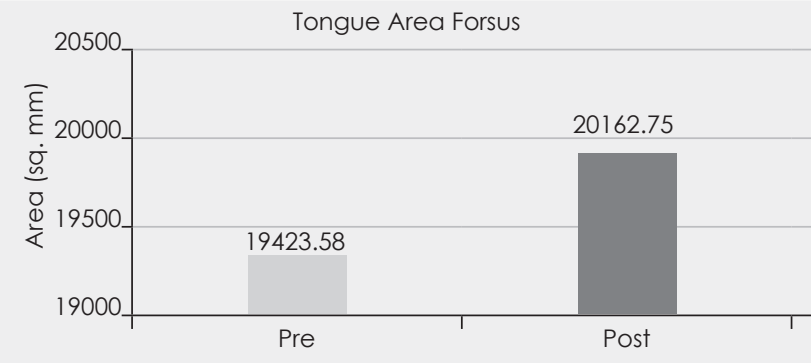

Graph 5: Comparison of mean of Tongue area in pre and post treatment of Forsus (Group B)

\section{DISCUSSION}

In Class II patients with retruded mandible, backward placement of mandible may lead to inferoposterior position of hyoid bone, posteriorly positioned tongue and soft palate which may increase the chances of

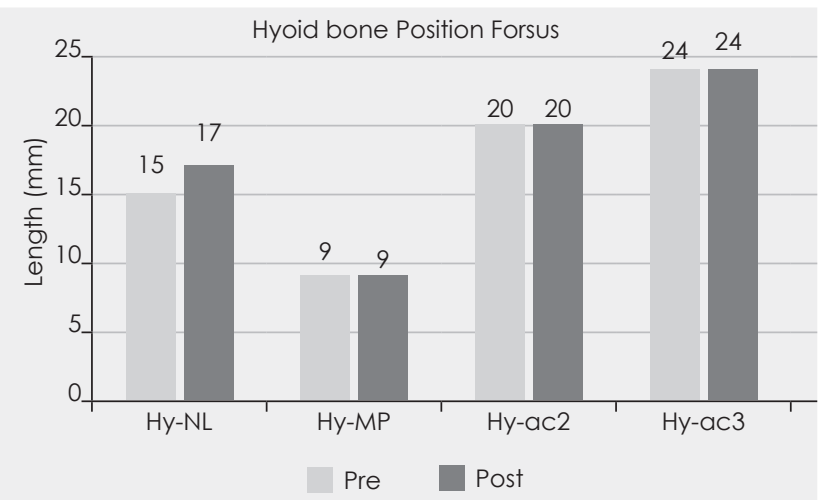

Graph 2: Comparison of mean of Hyoid Bone position in pre and post treatment of Forsus (Group B)

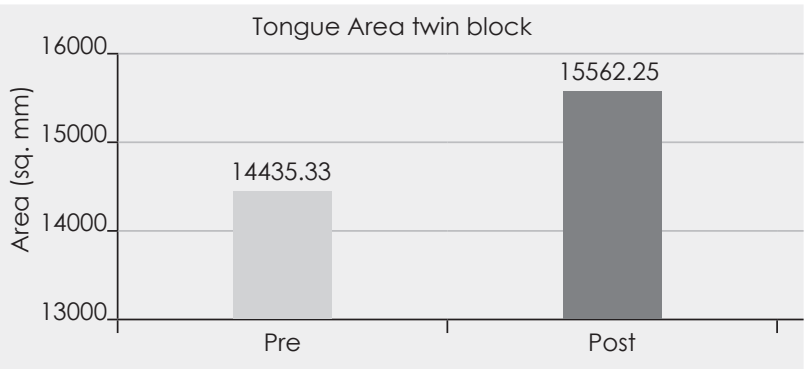

Graph 4: Comparison of mean of Tongue area in pre and post treatment of Twin Block (Group A)

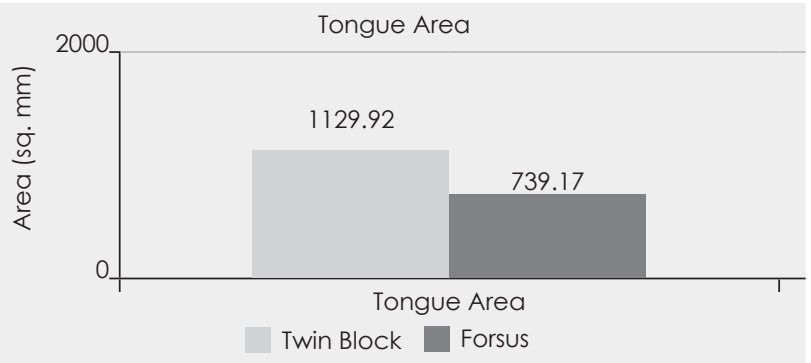

Graph 6: Twin Block (Group A) vs. Forsus (Group B) comparison of mean difference of Tongue area

impaired respiratory functions. The anteroposterior position of mandible has also effect on position of hyoid bone which is associated with important functions as deglutition, phonation, and respiration. Hoffman and Hoffman ${ }^{7}$ believed that the hyoid bone was important for tongue position, since most of extrinsic muscle of 
the tongue are attached to it and it also maintains the pharyngeal airway.

Bucchieri investigated that the altered hyoid bone position may influence the tongue position and upper airway patency. Hence, any change in position of mandible and hyoid bone can adversely affect the dimensions of airway. Grant' also reported difference in hyoid bone position, and found hyoid bone was higher in Class III than Class II subjects in relation to the cervical vertebrae. According to Thurow, ${ }^{10}$ the geniohyoid muscle functions to adjust the anteroposterior position of the hyoid and to maintain the airway patency throughout the various movements of the craniofacial complex.

According to the Balter's ${ }^{11}$ philosophy, Class II malocclusion are a consequence of backward position of a tongue, disturbing the cervical region. Thus, it is necessary to assess tongue in orthodontic diagnosis and treatment planning.

Peat ${ }^{12}$ postulated the role of tongue in positioning of dento-alveloar structures. Not only the function, but also the growth, posture or function of tongue is of significance. Rakosi ${ }^{13}$ proposed that abnormalities of either posture or function could possibly contribute to development of skeletal malocclusion.

Correction of retruded mandible with functional appliances also has positive effect on hyoid bone position and tongue area. Kalgotra ${ }^{14}$ stated that as the body of the mandible lengthens, the attachments of the genioglossus and geniohyoid muscles move forward away from the oropharynx, increasing the pharyngeal space and found significant positive correlation between tongue position and Class II skeletal patterns. This study evaluated \& compared hyoid bone position and tongue area changes with the Twin Block and Forsus appliances.

\section{Skeletal Parameters:}

The main skeletal change that results from our study is mandibular advancement. Nevertheless, this advancement is due to change in both mandibular length and effective mandibular length seen with both the appliances. A similar observations was made by Ghodke et $\mathrm{al}^{15}$ and Vinoth et al. $^{16}$ SNB angle showed change in pre and post treatment values of Twin Block and Forsus and was in accordance with those of Bidayet $\mathrm{al}^{1}{ }^{17}$ Vinoth et $\mathrm{al},{ }^{16}$ Elfeky et $\mathrm{al}^{1}{ }^{18}$ Ghodke et $\mathrm{al}^{15}$ and Jena et $\mathrm{al}^{19}$ whereas Mohamad et $\mathrm{al}^{20}$ found no significant change in the SNB angle after Forsus treatment. On comparison between the appliances no significant difference was seen. There was also increase in W and FMPA angle following treatment with both the appliances.

The so-called "head-gear effect" was seen with the Twin Block appliance with decreased value of the SNA angle demonstrating the inhibition in anterior development of the maxilla and posterior repositioning from cranial base. This result is in accord with Vinothet $\mathrm{al}^{16}$ study. No significant change in SNA angle with Forsus was seen. This result showed significant difference between both the appliances in SNA angle.

\section{Hyoid Bone Position}

In present study, the results showed a significant upward \& forward movement of hyoid bone following Twin Block \& Forsus treatment. Brodie ${ }^{21}$ brought attention to the suprahyoid muscles which suspend hyoid bone and tongue, since these muscles are attached to the symphysis of mandible, the hyoid bone passively follow the course of chin.

Intergroup comparison revealed insignificant results, showing that the upward and forward movement of hyoid bone achieved by the two appliances are similar and changes in the effective mandibular length following the functional appliance treatment was seen in both the groups. As the mandible was advanced a balance is restored between the suprahyoid and infrahyoid muscles and the hyoid bone moved both upward \& forward. Verma et $\mathrm{al}^{22}$ reported that under the influence of Twin Block appliance treatment hyoid bone shifted forward in horizontal dimension and upward direction in vertical dimension.

The study of Ozdemir ${ }^{23}$ found no change in hyoid bone position following Forsus which could be due to the fact that no skeletal effects on the mandible were reported. The Class II correction was achieved only by dentoalveolar changes, whereas in our study Class II correction was achieved with true skeletal changes (effective mandibular lengthening) following Forsus treatment.

In accordance with results, effect of Twin Block and Forsus, when compared they are insignificant, indicating similar effect on hyoid bone. This observation supported the concept that the hyoid bone moved in conjunction with adjacent anatomic structures when the mandible is advanced.

\section{Tongue Area}

Significant increase in tongue area was seen with both the appliances. This change in tongue area was a 
favourable outcome as the mandibular advancement has increased the space available for tongue area. The previous study done by Ozdemiret al ${ }^{23}$ also found the significant increase in tongue area following Forsus treatment.

The results show that effect of Twin Block on tongue area is more than Forsus, but when compared they are insignificant, indicating similar increase in tongue area with both appliance.

\section{CONCLUSION}

- Significant upward and forward position of hyoid bone with both appliances. However, inter-group comparison revealed insignificant results showing smililar effects.

- Tongue area increased following both Twin Block \& Forsus therapy. The intergroup comparison showed insignificant difference between both the appliances, indicating similar effect on tongue area.

\section{OJN}

\section{REFERENCES}

1. Proffit WR, Moray LJ. Prevalence of malocclusion and orthodontic treatment need in the United States. Int J Adult OrthodonOrthognathSurg 1998;13:97-106

2. Silva NN, Lacerda RH, Silva AW, Ramos TB. Assessment of upper airways measurements in patients with mandibular skeletal Class II malocclusion. Dental press journal of orthodontics. 2015 Oct;20(5):86-93.

3. Ozbek MM, Memikoglu UT, Gogen H, Lowe AA, Baspinar E. Oropharyngeal airway dimensions and functional orthopaedic treatment in skeletal class II cases. Angle Orthod. 1998;68:327-326.

4. Enache AM, Nimigean VR, Mihăltan F, Didilescu AC, Munteanu I, Nimigean V. Assessment of sagittal and vertical skeletal patterns in Romanian patients with obstructive sleep apnea. Rom J MorpholEmbryol. 2010 Jan 1;51 (3):505-8.

5. Khanna R, TikkU T, Sharma VP. Position and orientation of hyoid bone in class II división 1 subjects: A cephalometric study. J Indian Orthod Soc. 2011 Jan 1;45:212-8

6. Johal AM, Patel SI, Battagel JM. The relationship between craniofacial anatomy and obstructive sleep apnoea: a case-controlled study. Journal of sleep research. 2007 Sep; 16(3):319-26.

7. Hoffman JA, Hoffman RL. Tongue thurst and deglutition: Some anatomical, physiological and neurological considerations. J Speech Hear disorder , 1965;30:105-20.

8. Bucchieri A, Mastrangelo C, Stella R, Poladas EG. Cephalometric evaluation of hyoid bone position in patients with obstructive sleep apnea. Minerva stomatologica. 2004;53(1-2):33-9.

9. Grant LE. A radiographic study of the hyoid bone position in angle class I, II and III. Unpublished Master Thesis. University of Uanas city.

10. Kjellberg H. Juvenile chronic arthritis. Dentofacial morphology, growth, mandibular function and orthodontic treatment. Swed Dent J Suppl. 1995;109:1-56.

11. Graber TM, Neumann B. Bionator. In: Graber TM, Neumann B, editors. Removable Orthodontic Appliances. 2nd ed. Philadelphia: WB Saunders; $1984 ; 357-75$.

12. Peat JH. A cephalometric study of tongue position. American journal of orthodontics. 1968 May 1;54(5):339-51.

13. Rakosi T. London. An atlas and manual of cephalometric radiography. Wolf Medical Publication Limited; $1978: 96-8$.

14. Kalgotra S, Mushtaq M. Position of Tongue in skeletal Class II \& Class III-ACephalometric study.

15. SwapnilGhodke; Ashok Kumar Utreja; Satinder Pal Singh and Ashok Kumar Jena.Effect of Twin block applianc on the anatomy of the pharyngeal airway passage (PAP) in class II malocclusion subjects. Progress in Orthodontocs. 2014;15:68.

16. Vinoth SK, Thomas AV, Nethravathy R. Cephalomteric changes in airway dimensions with twin block therapy in growing Class II patients. Journal of pharmacy \&bioallied sciences. 2013 Jun;5(Suppl 1):S25.

17. Biday S, Chaudhari CV, Ramkrishna S. Skeletal And Dentoalveolar Changes Seen In Class II Div 1 Mal-Occlusion Cases Treated With Twin Block Appliance-A Cephalometric Study. IOSR Journal of Dental and Medical Sciences. 2014 Feb;13(1).

18. y Elfeky H, Fayed MM. Three-dimensional effects of twin block therapy on pharyngeal airway parameters in Class II malocclusion patients. Journal of the World Federation of Orthodontists. 2015 Sep 1;4(3):114-9.

19. Ashok Kumar Jena; Satinder Pal Singh; Ashok Kumar Utreja. Effectiveness of twin block and Mandibular protraction appliance in the improvement of pharyngeal airway passage dimension in class II malocclusion subjects with retrognathic mandible. Angle Orthod. 2013;83:728-734.

20. Mahamad IK, Neela PK, Mascarenhas R, Akhter Husain BD. A comparision of Twin-block and Forsus (FRD) functional appliance-a cephalometric study. IJO. 2012;23(3).

21. Brodie AG. Consideration of musculature in diagnosis, treatment, and retention. American Journal of Orthodontics. 1952 Nov 1;38(1 1):82335.

22. Verma G, Tandon P, Nagar A, Singh GP, Singh A. Cephalometric evaluation of hyoid bone position and pharyngeal spaces following treatment with Twin block appliance. Journal of orthodontic science. 2012 Jul; 1 (3):77.

23. FulyaOzdemir; FeyzaUlkar; DidemNalbantagil. Effects of fixed functional therapy on tongue and hyoid positions and posterior airway. Angle Orthd. 2014; 84:260-264. 